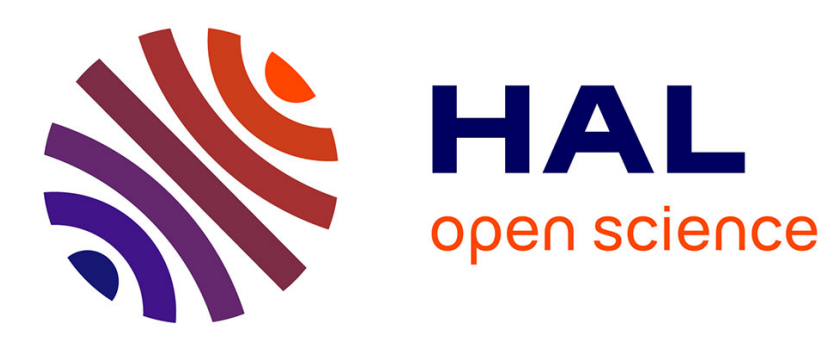

\title{
Open Loop Controllers to Solve the Disturbance Decoupling Problem for Max-Plus Linear Systems
}

Ying Shang, Laurent Hardouin, Mehdi Lhommeau, Carlos-Andrei Maia

\section{To cite this version:}

Ying Shang, Laurent Hardouin, Mehdi Lhommeau, Carlos-Andrei Maia. Open Loop Controllers to Solve the Disturbance Decoupling Problem for Max-Plus Linear Systems. European Control Conference, ECC 2013, 2013, Zurich, Switzerland. hal-01113450

\author{
HAL Id: hal-01113450 \\ https://hal.science/hal-01113450
}

Submitted on 5 Feb 2015

HAL is a multi-disciplinary open access archive for the deposit and dissemination of scientific research documents, whether they are published or not. The documents may come from teaching and research institutions in France or abroad, or from public or private research centers.
L'archive ouverte pluridisciplinaire HAL, est destinée au dépôt et à la diffusion de documents scientifiques de niveau recherche, publiés ou non, émanant des établissements d'enseignement et de recherche français ou étrangers, des laboratoires publics ou privés. 


\title{
Open Loop Controllers to Solve the Disturbance Decoupling Problem for Max-Plus Linear Systems
}

\author{
Ying Shang, Laurent Hardouin, Mehdi Lhommeau and Carlos Andrey Maia
}

\begin{abstract}
This paper presents the new investigations on the disturbance decoupling problem (DDP) in the geometric control of max-plus linear systems. Due to the monotone nondecreasing property of max-plus linear systems, a modified disturbance decoupling problem (MDDP) is defined and an optimal controller to solve the MDDP is obtained using a prefilter mapping by taking the disturbances into account. Moreover, this paper presents a necessary and sufficient condition such that the same optimal controller for the MDDP can be used to solve the DDP for max-plus linear systems. When the necessary and sufficient condition is not satisfied, a new disturbance mapping is developed in order to guarantee the solvability of the DDP by the same optimal controller for the MDDP. The main results of this paper are illustrated by using a timed event graph model of a manufacturing network.
\end{abstract}

\section{INTRODUCTION}

Max-plus linear systems ([1], [11], [14], [16]) have been studied in the past three decades, and many fundamental problems have been investigated by researchers, such as controllability [21], observability [12], and the model reference control problem [19]. A new research area for max-plus linear systems is to establish the geometric control theory [22] as predicted in [6]. There are some existing research results on generalizing fundamental concepts and problems in geometric control to max-plus linear systems, such as computations of different controlled invariant sets ([10], [15], [20]) and the disturbance decoupling problem [17].

This paper reports upon new investigations on the disturbance decoupling problem (DDP) for max-plus linear systems. For classical linear systems, the DDP means that the output signals remain unchanged in the presence of the disturbances. Due to the monotone nondecreasing property of max-plus linear systems, the controller can only increase the inputs, in other words, it is only possible to delay the input tokens in the corresponding timed event graph. From a practical point of view, it would be interesting to ask the question as whether there exists a controller such that the system is not disturbed more than the disturbances have. For example, when a system break down occurs,

Y. Shang is with the Department of Electrical and Computer Engineering, Southern Illinois University Edwardsville, Campus Box 1801, Edwardsville, IL 62026, USA yshang@ siue. edu

L. Hardouin and M. Lhommeau are with the Laboratoire d'Ingénierie des Systèmes Automatisés of the University of Angers, 62 avenue Notre Dame du Lac, 49000 Angers, France laurent.hardouinduniv-angers.fr, and mehdi. lhommeau@univ-angers. fr, respectively.

C.A. Maia is with Departamento de Engenharia Elétrica, Universidade Federal de Minas Gerais (UFMG). Av. Antônio Carlos 6627, Pampulha, 31270-010, Belo Horizonte, MG, Brazil maia@ cpdee. ufmg.br and was supported by the Brazilian Agencies CAPES, CNPQ and FAPEMIG. we can put the input parts of the manufacturing line as late as possible to reduce the unnecessary waiting time in the network. Therefore, a so called modified disturbance decoupling problem (MDDP) is defined in this paper and an optimal controller for the MDDP is obtained using a prefilter mapping. Moreover, this paper presents a necessary and sufficient condition such that the same optimal controller for the MDDP can be used to solve the DDP for max-plus linear systems. This condition requires the output image of the reachable space of the disturbances has to be a subset of the output image of the reachable space of the controls. When the necessary and sufficient condition is not satisfied, a new disturbance mapping is developed in order to guarantee the solvability of the DDP using the same optimal controller for the MDDP. The main results are illustrated using a timed event graph model of a manufacturing network.

The remainder of this paper is organized as follows. Section II presents the mathematical preliminaries in the max-plus algebra literature. Section III presents the maxplus linear system models. Section IV defines the concepts of the DDP and MDDP, and establishes the optimal controller to solve the MDDP. Section V establishes the relationships between the optimal controller for the MDDP and the solution to the DDP for max-plus linear systems. Section VI is the illustration using a timed event graph model for a manufacturing system. Section VII concludes this paper.

\section{Mathematical Preliminaries}

A semiring is a set $\mathcal{S}$, equipped with two operations $\oplus, \otimes$, such that $(\mathcal{S}, \oplus)$ is a commutative monoid (the zero element will be denoted $\varepsilon),(\mathcal{S}, \otimes)$ is a monoid (the unit element will be denoted $e$ ), operation $\otimes$ is right and left distributive over $\oplus$, and $\varepsilon$ is absorbing for the product (i.e. $\varepsilon \otimes a=a \otimes \varepsilon=$ $\varepsilon, \forall a$ ). A semiring $\mathcal{S}$ is idempotent if $a \oplus a=a$ for all $a \in \mathcal{S}$. A non empty subset $\mathcal{B}$ of a semiring $\mathcal{S}$ is a subsemiring of $\mathcal{S}$ if for all $a, b \in \mathcal{B}$ we have $a \oplus b \in \mathcal{B}$ and $a \otimes b \in \mathcal{B}$. In this paper, we denote $\overline{\mathbb{Z}}_{\max }=(\mathbb{Z} \cup\{-\infty,+\infty\}, \max ,+)$ as the integer max-plus semiring.

In an idempotent semiring $\mathcal{S}$, operation $\oplus$ induces a partial order relation

$$
a \succeq b \quad \Longleftrightarrow \quad a=a \oplus b, \quad \forall a, b \in \mathcal{S} .
$$

Then, $a \vee b=a \oplus b$. We say that an idempotent semiring $\mathcal{S}$ is complete if it is complete as an ordered set, and if for all $a \in \mathcal{S}$, the left and right multiplications ${ }^{1}$ by $a, L_{a}$ :

\footnotetext{
${ }^{1}$ The symbol $\otimes$ is often omitted.
} 
$\mathcal{S} \rightarrow \mathcal{S}, x \mapsto a x$ and $R_{a}: \mathcal{S} \rightarrow \mathcal{S}, x \mapsto x a$ are lower semicontinuous. A map $L_{a}: \mathcal{S} \rightarrow \mathcal{S}$ is lower semi-continuous if $L_{a}\left(\oplus_{x \in \mathcal{S}} x\right)=\oplus_{x \in \mathcal{S}} L_{a}(x)$. These maps are residuated, i.e. $L_{a}(x) \preceq b$ (resp. $R_{a}(x) \preceq b$ ) admits a greatest solution , then the following notations are considered :

$$
\begin{aligned}
& L_{a}^{\sharp}(b)=a \phi b=\bigoplus\{x \mid a x \preceq b\} \text { and } \\
& R_{a}^{\sharp}(b)=b \phi a=\bigoplus\{x \mid x a \preceq b\}, \quad \forall a, b \in \mathcal{S} .
\end{aligned}
$$

where $L_{a}^{\sharp}$ is called the residual mapping, and is the unique mapping such that $L_{a} \circ L_{a}^{\sharp} \preceq I d$ and $L_{a}^{\sharp} \circ L_{a} \succeq I d$ where $I d$ is the identity mapping (the same holds for $R_{a}$ ). The implicit equation $x=a x \oplus b$ admits $x=a^{*} b=\left(\bigoplus_{k>0} a^{k}\right) b$ as smallest solution. All these results admit a natural extension to the matrix case, where the sum and product of matrices are defined with the same rules as in classical theory (see [1]).

Definition 1 ([4], [5], [7]): Let $\mathcal{S}$ be a complete idempotent semiring and let $C$ be a $n \times p$ matrix with entries in $\mathcal{S}$. We call null kernel of $C$ as the set of elements $x \in \mathcal{S}^{p}$ such that $C x=\epsilon$, denoted as $\operatorname{ker} C$. We call equivalence kernel of $L_{C}$ (denoted by $\operatorname{ker}_{\mathrm{eq}} C$ ), the subset of all pairs of elements of $\mathcal{S}^{p}$ whose components are both mapped by $L_{C}$ to the same element in $\mathcal{S}^{n}$, i.e., the following definition

$$
\operatorname{ker}_{\mathrm{eq}} C:=\left\{\left(s, s^{\prime}\right) \in\left(\mathcal{S}^{p}\right)^{2} \mid C s=C s^{\prime}\right\} .
$$

Clearly $\operatorname{ker}_{\text {eq }} C$, is an equivalence relation on $\mathcal{X}$, i.e., $C s=$ $C s^{\prime} \Longleftrightarrow s^{\prime} \equiv s\left(\operatorname{mod~ker~}_{\mathrm{eq}} C\right)$ and furthermore it is a congruence and then we can define the quotient $\mathcal{S} / \operatorname{ker}_{e q} C$.

The subset of elements $s^{\prime} \in \mathcal{S}^{p}$ that are equivalent to $s$ modulo $\operatorname{ker}_{e q} C$ is denoted $[s]_{C}$, i.e.,

$$
[s]_{C}=\left\{s^{\prime} \in \mathcal{S}^{p} \mid s^{\prime} \equiv s\left(\bmod \operatorname{ker}_{\mathrm{eq}} C\right)\right\} \subset \mathcal{S}^{p} .
$$

\section{MAX-Plus Linear Systems}

A general max-plus linear model with disturbances is defined as

$$
\begin{aligned}
x(k)= & \bigoplus_{i=0}^{a} A_{i} x(k-i) \oplus \bigoplus_{j=0}^{b} B_{j} u(k-j) \\
& \oplus \bigoplus_{l=0}^{c} S_{l} q(k-l), \\
y(k)= & \bigoplus_{r=0}^{d} C_{r} x(k-r),
\end{aligned}
$$

where $x(k) \in X \cong \overline{\mathbb{Z}}_{\text {max }}^{n}, u(k) \in U \cong \overline{\mathbb{Z}}_{\text {max }}^{p}, q(k) \in Q \cong \overline{\mathbb{Z}}_{\text {max }}^{r}$, $y(k) \in Y \cong \overline{\mathbb{Z}}_{\max }^{q}$ and $k \in \mathbb{Z}$. After some modifications [3], it is possible to obtain the following explicit form:

$$
\begin{aligned}
x(k) & =A_{0} x(k) \oplus A_{1} x(k-1) \oplus B_{0} u(k) \oplus S_{0} q(k), \\
y(k) & =C x(k) .
\end{aligned}
$$

Furthermore, by solving the implicit equation, the equation above can be written as

$$
\begin{aligned}
& x(k)=A_{0}^{*} A_{1} x(k-1) \oplus A_{0}^{*} B_{0} u(k) \oplus A_{0}^{*} S_{0} q(k), \\
& x(k)=A x(k-1) \oplus B u(k) \oplus S q(k), \\
& y(k)=C x(k),
\end{aligned}
$$

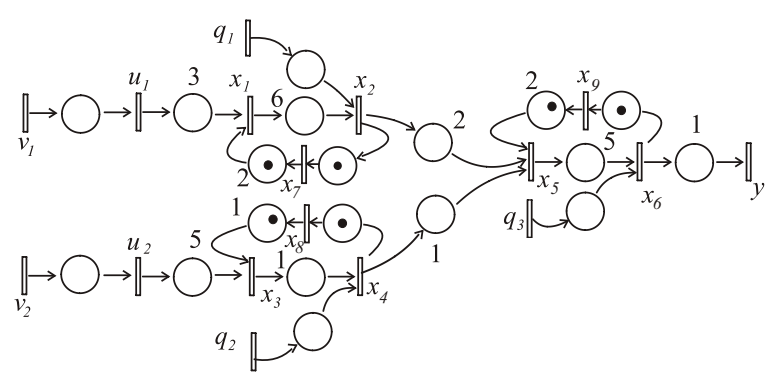

Fig. 1. The timed event graph model of a manufacturing system.

where $A_{0}^{*}=\bigoplus_{i \in \mathbb{N}} A_{0}^{i}$ is with finite entries if the corresponding graph of this matrix is without circuit.

For the timed event graph of a manufacturing line shown in Fig. 1, the max-plus linear system equations are described as the following:

$$
\begin{aligned}
& x_{1}(k)=2 x_{7}(k-1) \oplus 3 u_{1}(k) \\
& x_{2}(k)=6 x_{1}(k) \oplus 0 q_{1}(k) \\
& x_{3}(k)=1 x_{8}(k-1) \oplus 5 u_{2}(k) \\
& x_{4}(k)=1 x_{3}(k) \oplus 0 q_{2}(k) \\
& x_{5}(k)=2 x_{2}(k) \oplus 1 x_{4}(k) \oplus 2 x_{9}(k-1) \\
& x_{6}(k)=5 x_{5}(k) \oplus 0 q_{3}(k) \\
& x_{7}(k)=0 x_{2}(k-1) \\
& x_{8}(k)=0 x_{4}(k-1) \\
& x_{9}(k)=0 x_{6}(k-1) .
\end{aligned}
$$

The corresponding max-plus linear system matrices $A_{0}, A_{1}$, $B_{0}, S_{0}$ and $C$ in Eq. (4) have null entries except for the following ones

$$
\begin{aligned}
& A_{0}(2,1)=6, A_{0}(4,3)=1, A_{0}(5,2)=2, A_{0}(5,4)=1, \\
& A_{0}(6,5)=5, \\
& A_{1}(1,7)=2, A_{1}(3,8)=1, A_{1}(5,9)=2, A_{1}(7,2)=0, \\
& A_{1}(8,4)=0, A_{1}(9,6)=0, \\
& B_{0}(1,1)=3, B_{0}(3,2)=5 \\
& S_{0}(2,1)=e, S_{0}(4,2)=e, S_{0}(6,3)=e \\
& C(1,6)=1 .
\end{aligned}
$$

After computation of $A_{0}^{*}$, matrices $A, B, S$ are obtained with the following non null entries:

$$
\begin{aligned}
& A(1,7)=2, A(2,7)=8, A(3,8)=0, A(4,8)=2, \\
& A(5,7)=10, A(5,8)=3, A(5,9)=2, A(6,7)=15 \\
& A(6,8)=8, A(6,9)=7, A(7,2)=e, A(8,4)=e \\
& A(9,6)=e \\
& B(1,1)=3, B(2,1)=9, B(3,2)=5, B(4,2)=6 \\
& B(5,1)=11, B(5,2)=7, B(6,1)=16, B(6,2)=12, \\
& S(2,1)=e, S(4,2)=e, S(5,1)=2, S(5,2)=1 \\
& S(6,1)=7, S(6,2)=6, S(6,3)=e .
\end{aligned}
$$

This kind of system makes it possible to describe the behaviors of timed event graphs, which is a subclass of timed Petri net such that each place has one and only one input and output transition (see Fig. 1). By associating to each transition $x_{i}$ a firing date sequence $x_{i}(k) \in \mathbb{Z}$, it is possible to predict the system evolution thanks to equation (4). For each increasing sequence $\{x(k)\}$, it is possible to define the transformation $X(\gamma)=\bigoplus_{k \in \mathbb{Z}} x(k) \gamma^{k}$ where $\gamma$ is a backward shift operator in event domain (i.e., $Y(\gamma)=\gamma X(\gamma) \Longleftrightarrow$ $\{y(k)\}=\{x(k-1)\}$, (see [1], p. 228). This transformation is 
analogous to the $Z$-transform used in discrete-time classical control theory and the formal series $X(\gamma)$ is a synthetic representation of the trajectory $x(k)$. The set of the formal power series in $\gamma$ is denoted by $\overline{\mathbb{Z}}_{\max } \llbracket \gamma \rrbracket$ and constitutes an idempotent semiring. Therefore, the state equation in Eq. (4) becomes a polynomial equation or a frequency domain representation,

$$
\begin{aligned}
X(\gamma) & =\left(A_{0} \oplus \gamma A_{1}\right) X(\gamma) \oplus x_{0} \oplus B_{0} U(\gamma) \oplus S_{0} Q(\gamma) \\
& =\bar{A} X(\gamma) \oplus x_{0} \oplus B_{0} U(\gamma) \oplus S_{0} Q(\gamma) \\
Y(\gamma) & =C X(\gamma)
\end{aligned}
$$

for any initial state $x_{0}$, assuming $u(0)=q(0)=\epsilon$, the state $X(\gamma) \in \mathcal{X}=\left(\overline{\mathbb{Z}}_{\max } \llbracket \gamma \rrbracket\right)^{n \times 1}$, the output $Y(\gamma) \in \mathcal{Y}=$ $\left(\overline{\mathbb{Z}}_{\max } \llbracket \gamma \rrbracket\right)^{q \times 1}$, the input $U(\gamma) \in \mathcal{U}=\left(\overline{\mathbb{Z}}_{\max } \llbracket \gamma \rrbracket\right)^{p \times 1}$, and the disturbance $Q(\gamma) \in \mathcal{Q}=\left(\overline{\mathbb{Z}}_{\max } \llbracket \gamma \rrbracket\right)^{r \times 1}$, and matrices $\bar{A} \triangleq A_{0} \oplus \gamma A_{1} \in\left(\overline{\mathbb{Z}}_{\max } \llbracket \gamma \rrbracket\right)^{n \times n}, B_{0} \in\left(\overline{\mathbb{Z}}_{\max } \llbracket \gamma \rrbracket\right)^{n \times p}, C \in$ $\left(\overline{\mathbb{Z}}_{\max } \llbracket \gamma \rrbracket\right)^{q \times n}$ and $S_{0} \in\left(\overline{\mathbb{Z}}_{\max } \llbracket \gamma \rrbracket\right)^{n \times r}$ represent the link between transitions. The trajectories $U(\gamma)$ and $Y(\gamma)$ can be related ([1], p. 243) by the equation $Y(\gamma)=H(\gamma) U(\gamma)$, where $H(\gamma)=C \bar{A}^{*} B_{0} \in\left(\overline{\mathbb{Z}}_{\max } \llbracket \gamma \rrbracket\right)^{q \times p}$ is called the transfer matrix of the TEG. Entries of matrix $H$ are periodic series ([1], p. 260) in the idempotent semiring, usually represented by $p(\gamma) \oplus q(\gamma)\left(\tau \gamma^{\nu}\right)^{*}$, where $p(\gamma)$ is a polynomial representing the transient behavior, $q(\gamma)$ is a polynomial corresponding to a pattern which is repeated periodically, the period being given by the monomial $\left(\tau \gamma^{\nu}\right)$. The disturbances are uncontrollable inputs acting on the system's internal state ${ }^{2}$, through matrix $S_{0}$, and $C \bar{A}^{*} S_{0} \in\left(\overline{\mathbb{Z}}_{\max } \llbracket \gamma \rrbracket\right)^{q \times r}$ is the transfer function between the disturbances and outputs.

\section{Disturbance Decoupling of MaX-Plus Linear SYSTEMS}

\section{A. Disturbance Decoupling Problem}

For max-plus linear systems in Eq. (5), we introduce the definition of the disturbance decoupling problem (DDP):

Definition 2: The system (5) is called disturbance decoupled by an open-loop controller $u(k)$ if and only if any disturbance signal will not affect the system output $y(k)$ for all $k \in \mathbb{Z}$ and for any initial condition $x_{0}$.

Proposition 1: ([13]) Given a max-plus linear system of the form (5), the DDP is solvable by an open-loop controller $u(k)$ if and only if there exists an open-loop control sequence $\vec{u}=\left[\begin{array}{llll}u(n) & u(n-1) & \cdots & u(1)\end{array}\right]^{T}$ such that the equivalence relation holds

$$
\langle A \mid B\rangle_{n} \vec{u} \oplus\langle A \mid S\rangle_{n} \vec{q} \equiv_{\operatorname{ker}_{\mathrm{eq}} C}\langle A \mid B\rangle_{n} \vec{u}
$$

where

$$
\langle A \mid S\rangle_{n}=\left[\begin{array}{llll}
S & A S & \cdots & A^{(n-1)} S
\end{array}\right]
$$

and

$$
\langle A \mid B\rangle_{n}=\left[\begin{array}{llll}
B & A B & \cdots & A^{(n-1)} B
\end{array}\right],
$$

for all $n$ and any disturbance signal

$$
\vec{q}=\left[\begin{array}{llll}
q(n) & q(n-1) & \cdots & q(1)
\end{array}\right]^{T} .
$$

\footnotetext{
${ }^{2}$ They model event blocking the system, e.g. machine breakdown, uncontrollable component supply...
}

Proposition 1 indicates that, in order to check the solvability condition for the DDP of max-plus linear systems, we need an infinite number of checking for the equation (7). The computational complexity of this result can be resolved by the $\gamma$-domain analysis approach instead of the event domain analysis approach, which will be discussed in details later.

\section{B. Modified Disturbance Decoupling Problem (MDDP)}

Model (5) describes the fastest behavior of the system, hence the control $u$ only makes possible to increase the inputs, in other words, it is only possible to delay the input of tokens in the corresponding timed event graph. In the same way, the uncontrollable disturbance input $q$ acts by delaying the firing of the transitions. Hence, it could be interesting to delay the input $u$ as much as possible in order to match this disturbance. From a practical point of view, this means that the token will be put in the system as late as possible in order not to disturb the system more than the disturbances have, hence the strategy will reduce the useless waiting time as much as possible. In summary, this strategy is described in the following definition:

Definition 3: The max-plus linear system described in Eq. (5) is called modified disturbance decoupled by an openloop controller $u(k)$ if and only if the system output signals will not be disturbed more than the disturbances have.

\section{MDDP with an Open-Loop Controller}

The objective of the MDDP using an open-loop controller is to find an open-loop controller $U(\gamma)$ such that, for any initial condition $x_{0}$ in the state space, the system output will not be disturbed more than the disturbance signal has. Instead of using the event domain analysis for Eq. (4) and Eq. (5), we obtain the system trajectories for Eq. (6) in the $\gamma$-domain for any initial condition $x_{0}$ :

$$
\begin{aligned}
X(\gamma) & =\bar{A}^{*} B_{0} U(\gamma) \oplus \bar{A}^{*} S_{0} Q(\gamma) \oplus \bar{A}^{*} x_{0} \\
& =\bar{A}^{*}\left[B_{0} \mid S_{0}\right]\left(\begin{array}{l}
U(\gamma) \\
Q(\gamma)
\end{array}\right) \oplus \bar{A}^{*} x_{0} \\
& =\bar{A}^{*} \widetilde{B}\left(\begin{array}{l}
U(\gamma) \\
Q(\gamma)
\end{array}\right) \oplus \bar{A}^{*} x_{0} \\
Y(\gamma) & =C \bar{A}^{*} B_{0} U(\gamma) \oplus C \bar{A}^{*} S_{0} Q(\gamma) \oplus C \bar{A}^{*} x_{0} \\
& =C \bar{A}^{*} \widetilde{B}\left(\begin{array}{l}
U(\gamma) \\
Q(\gamma)
\end{array}\right) \oplus C \bar{A}^{*} x_{0} .
\end{aligned}
$$

In order to solve for the MDDP using an open-loop controller, then according to Definition 3 , the following equality has to hold for any initial condition:

$$
\begin{aligned}
C \bar{A}^{*} \widetilde{B}\left(\begin{array}{l}
U(\gamma) \\
Q(\gamma)
\end{array}\right) \oplus C \bar{A}^{*} x_{0}=C \bar{A}^{*} S_{0} Q(\gamma) \oplus C \bar{A}^{*} x_{0}, \forall x_{0} \\
\Longleftrightarrow C \bar{A}^{*} \widetilde{B}\left(\begin{array}{l}
U(\gamma) \\
Q(\gamma)
\end{array}\right)=C \bar{A}^{*} S_{0} Q(\gamma)
\end{aligned}
$$

In other words, the objective of the MDDP by an openloop controller is to characterize the greatest state $X(\gamma) \in$ $\operatorname{Im} \bar{A}^{*} \widetilde{B}=\left\{\bar{A}^{*} \widetilde{B}\left(\begin{array}{l}U(\gamma) \\ Q(\gamma)\end{array}\right) \mid(U(\gamma), Q(\gamma)) \in \mathcal{U} \times \mathcal{Q}\right\}$ such that

$$
\begin{aligned}
& C X(\gamma)=C \bar{A}^{*} S_{0} Q(\gamma), \forall Q(\gamma) \in \mathcal{Q} \Longleftrightarrow \\
& \left(X(\gamma), \bar{A}^{*} S_{0} Q(\gamma)\right) \in \operatorname{ker}_{\mathrm{eq}} C, \forall Q(\gamma) \in \mathcal{Q}
\end{aligned}
$$


This is the greatest state $X(\gamma) \in \operatorname{Im} \bar{A}^{*} \widetilde{B}$ ensuring that the output $Y(\gamma)$ is equal to the one due to the disturbances. The solvability of the MDDP is not an issue because $U(\gamma)=$ $\epsilon$ is a trivial solution to Eq. (10). The solution set of all control inputs can also be upper bounded since the following equivalences hold :

$$
\begin{array}{ll}
C \bar{A}^{*} \widetilde{B}\left(\begin{array}{l}
U(\gamma) \\
Q(\gamma)
\end{array}\right) & =C \bar{A}^{*} S_{0} Q(\gamma) \\
\Longleftrightarrow C \bar{A}^{*} B_{0} U(\gamma) & \preceq C \bar{A}^{*} S_{0} Q(\gamma) \\
\Longleftrightarrow U(\gamma) & \preceq\left(C \bar{A}^{*} B_{0}\right) \phi\left(C \bar{A}^{*} S_{0} Q(\gamma)\right)=U(\gamma)_{o p t}^{Q} .
\end{array}
$$

Therefore the solutions set is an interval $\left[\varepsilon, U(\gamma)_{o p t}^{Q}\right]$, and the upper bound is the optimal solution according to the justin-time criterion, i.e. the greatest control that can reduce the useless waiting time in the system as much as possible.

However, this optimal controller $U(\gamma)_{o p t}^{Q}$ depends on the choice of the disturbance $Q(\gamma)$. If we have some degree of measurement of any disturbance $Q(\gamma)$, we can consider a control strategy as depicted in Fig. 2, where $P$ is a prefilter which generates the control by taking the disturbances into account. The resulting control $U(\gamma)=V(\gamma) \oplus P Q(\gamma)$ leads to the following output

$$
Y(\gamma)=C \bar{A}^{*} B_{0} P Q(\gamma) \oplus C \bar{A}^{*} B_{0} V(\gamma) \oplus C \bar{A}^{*} S_{0} Q(\gamma)
$$

and the problem consists of finding a controller $P$ such that:

$$
\begin{aligned}
Y(\gamma) & =C \bar{A}^{*} B_{0} P Q(\gamma) \oplus C \bar{A}^{*} B_{0} V(\gamma) \oplus C \bar{A}^{*} S_{0} Q(\gamma) \\
& \preceq C \bar{A}^{*} S_{0} Q(\gamma) \oplus C \bar{A}^{*} B_{0} V(\gamma), \forall V(\gamma), Q(\gamma) .
\end{aligned}
$$

where the right hand term of the inequality is the output without controller. This problem is equivalent to find a controller $P$ such that :

$$
C \bar{A}^{*} B_{0} P \preceq C \bar{A}^{*} S_{0}
$$

As previously a trivial lower bound to the solutions set is obtained by considering $V(\gamma)=\varepsilon$ and an optimal upper bound is given by

$$
P \preceq C \bar{A}^{*} B_{0} \phi C \bar{A}^{*} S_{0} \triangleq P_{o p t} .
$$

The optimal control mapping $P_{\text {opt }}$ is independent of any choice of $Q(\gamma)$, furthermore, the resulted optimal controller $P_{\text {opt }} Q(\gamma)$ can solve the MDDP for any given disturbance $Q(\gamma)$.

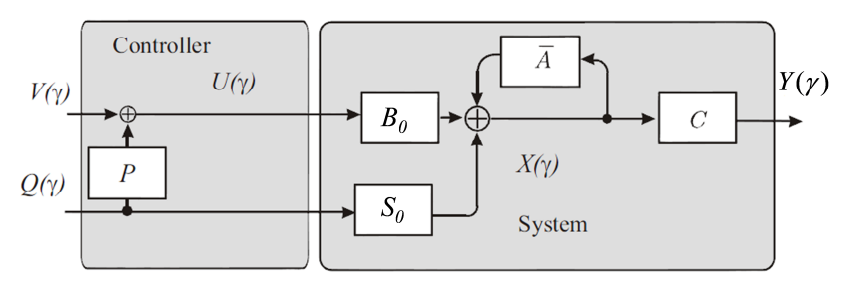

Fig. 2. The controller structure for MDDP.

\section{RELATIONSHIP BETWEEN MDDP AND DDP BY OPEN-LOOP CONTROLLERS}

The MDDP is always solvable because the control signals can be chosen to be zero to achieve the solvability. Our interest is whether a link can be established between the solutions to the MDDP and the solutions to the DDP of max-plus linear systems.

\section{A. Relationship between $P_{o p t}$ and DDP}

The question is whether such an optimal solution $P_{\text {opt }} Q(\gamma)$ will be able to solve the DDP of max-plus linear systems. We can have the following necessary and sufficient condition for such a connection.

Theorem 1: The optimal mapping $P_{\text {opt }}$ to the MDDP also solves the DDP for the max-plus linear systems described in Eq. (6) if and only if $\operatorname{Im} C \bar{A}^{*} S_{0} \subset \operatorname{Im} C \bar{A}^{*} B_{0}$.

Proof: "£ Sufficiency": If the condition $\operatorname{Im} C \bar{A}^{*} S_{0} \subset$ Im $C \bar{A}^{*} B_{0}$ holds, i.e., if $\exists L$ such that $C \bar{A}^{*} S_{0}=C \bar{A}^{*} B_{0} L$ then the optimal mapping $P_{o p t}$ satisfies the following equality

$$
\begin{aligned}
& \left.C \bar{A}^{*} B_{0} P_{o p t}=C \bar{A}^{*} B_{0}\left(\left(C \bar{A}^{*} B_{0}\right) \phi C \bar{A}^{*} B_{0} L\right)\right) \\
& =C \bar{A}^{*} B_{0} L=C \bar{A}^{*} S_{0},
\end{aligned}
$$

which implies

$$
\begin{aligned}
& C \bar{A}^{*} B_{0} P_{o p t} Q(\gamma) \oplus C \bar{A}^{*} S_{0} Q(\gamma) \oplus C \bar{A}^{*} B_{0} V(\gamma) \\
& =C \bar{A}^{*} B_{0} P_{\text {opt }} Q(\gamma) \oplus C \bar{A}^{*} B_{0} V(\gamma) .
\end{aligned}
$$

Equality in (14) means that the same optimal controller $P_{\text {opt }} Q(\gamma)$ can also solve the DDP in Definition 2 for any given disturbance $Q(\gamma)$, which means that the disturbance will not affect the system output.

" $\Longrightarrow$ Necessity": If the optimal mapping $P_{o p t}$ provides the optimal solution $P_{o p t} Q(\gamma)$ to the MDDP for any disturbance signal $Q(\gamma)$, then

$$
\begin{aligned}
& C A^{*} B_{0} P_{o p t} Q(\gamma) \oplus C \bar{A}^{*} S_{0} Q(\gamma) \oplus C \bar{A}^{*} B_{0} V(\gamma) \\
& =C \bar{A}^{*} B_{0} Q(\gamma) \oplus C \bar{A}^{*} B_{0} V(\gamma) .
\end{aligned}
$$

If the DDP is solvable at the same time by $P_{o p t}$, it implies that

$$
\begin{aligned}
& C \bar{A}^{*} B_{0} P_{o p t} Q(\gamma) \oplus C \bar{A}^{*} S_{0} Q(\gamma) \oplus C \bar{A}^{*} B_{0} V(\gamma) \\
& =C \bar{A}^{*} B_{0} P_{o p t} Q(\gamma) \oplus C \bar{A}^{*} B_{0} V(\gamma) .
\end{aligned}
$$

So the DDP is solvable by $P_{o p t}$ implies the equality of

$$
C \bar{A}^{*} B_{0} P_{o p t} Q(\gamma)=C \bar{A}^{*} S_{0} Q(\gamma) .
$$

Therefore, we have $\operatorname{Im} C \bar{A}^{*} S_{0} \subset \operatorname{Im} C \bar{A}^{*} B_{0} . \diamond$

\section{B. Relationship with DDP of Traditional Linear Systems}

We are interested in how Theorem 1's condition Im $C \bar{A}^{*} S_{0} \subset \operatorname{Im} C \bar{A}^{*} B_{0}$ is related to the solvability conditions of DDP for traditional linear systems over a field, described by the following equations:

$$
\begin{aligned}
& x(k)=A x(k-1)+B u(k)+S q(k) \\
& y(k)=C x(k),
\end{aligned}
$$

where $x(k) \in X \cong \mathbb{R}^{n}, u(k) \in U \cong \mathbb{R}^{p}, q(k) \in Q \cong \mathbb{R}^{r}$, $y(k) \in Y \cong \mathbb{R}^{q}$, and $k \in \mathbb{Z}, \mathbb{R}$ is the set of real numbers.

Proposition 2: For a traditional linear systems over a field in Eq. (15), the DDP is solvable by an open-loop controller if and only if $C\langle A \mid S\rangle=0$ or $\langle A \mid S\rangle \subset \operatorname{ker} C$, where $\langle A \mid S\rangle=$ $\left.\operatorname{Im} S+A \operatorname{lm} S \cdots+A^{(n-1)} \operatorname{Im} S\right)$. 
Proposition 3: For a traditional linear systems over a field in Eq. (15), the DDP is solvable by an open-loop controller implies $C\langle A \mid S\rangle \subset C\langle A \mid B\rangle$, where $\langle A \mid S\rangle=\operatorname{Im} S+A \operatorname{lm} S+$ $\cdots+A^{(n-1)} \operatorname{Im} S$ ), and $\langle A \mid B\rangle=\operatorname{Im} B+A \operatorname{lm} B+\cdots+$ $\left.A^{(n-1)} \operatorname{Im} B\right)$.

This means that the analogical condition $C\langle A \mid S\rangle \subset$ $C\langle A \mid B\rangle$ of Theorem 1's condition $\operatorname{Im} C \bar{A}^{*} S_{0} \subset \operatorname{Im} C \bar{A}^{*} B_{0}$ is only a necessary condition for the solvability of the DDP in the traditional case. Notice that the DDP for traditional linear systems require the control signals on both sides of the equation to be the same. In other words, for the same control, the outputs with or without the disturbances need to be the same in order to solve the DDP.

Here we introduce a so called output matching problem (OMP) in this paper as the following, if we can find a controller for a system with disturbances such that the output trajectory produced by a control and any disturbance remains the same from one original output without disturbances, but produced by a possibly different control, then any disturbance signals will still have no effect on the system outputs, similarly as the DDP. In another words, the set of the output trajectories remains invariant with or without the disturbances.

Proposition 4: For a traditional linear systems over a field in Eq. (15), the output matching problem is solvable by an open-loop controller if and only if $C\langle A \mid S\rangle \subset C\langle A \mid B\rangle$, where $\langle A \mid S\rangle=\operatorname{Im}\left(S+A S \cdots+A^{(n-1)} S\right)$, and $\langle A \mid B\rangle=\operatorname{Im}(B+$ $\left.A B \cdots+A^{(n-1)} B\right)$.

Therefore, Proposition 4 links the similar necessary and sufficient condition for the solvability of the output matching problem in the traditional linear systems as Theorem 1 for max-plus linear systems.

\section{Searching for Appropriate Disturbances to Solve DDP}

If the condition $\operatorname{Im} C \bar{A}^{*} S_{0} \subset \operatorname{Im} C \bar{A}^{*} B_{0}$ in Theorem 1 is not satisfied, then the optimal solution $P_{o p t}=$ $C \bar{A}^{*} B_{0} \phi C \bar{A}^{*} S_{0}$ to the MDDP will not be able to solve the DDP. The next reasonable question is to ask what type of disturbances will allow us to solve the DDP using the same optimal controller $P_{o p t}$ of the MDDP. In other words, for any disturbance $Q(\gamma)$, we need to achieve the equality (14). Our proposed approach is that, instead of keeping the original matrix $S_{0}$ which will not solve the DDP by $P_{o p t}$, we can try to find a matrix $\widetilde{S}_{0}$ such that the DDP be solved.

Lemma 1: Matrix

$$
\widetilde{S}_{0}^{o p t} \triangleq C \bar{A}^{*} \phi\left(C \bar{A}^{*} B_{0} P_{o p t}\right) \text {. }
$$

achieves equality $C \bar{A}^{*} B_{0} P_{\text {opt }}=C \bar{A}^{*} \widetilde{S}_{0}^{\text {opt }}$.

Proof: First, according to the residuation definition, the matrix $\widetilde{S}_{0}^{\text {opt }}$ is the greatest matrix such that $C \bar{A}^{*} \widetilde{S}_{0}^{\text {opt }} \preceq$ $C \bar{A}^{*} B_{0} P_{\text {opt }}$. On the other hand, due to the property of $L_{a}^{\sharp} \circ L_{a} \succeq I d$, we have $\widetilde{S}_{0}^{\text {opt }}=C \bar{A}^{*} \phi\left(C \bar{A}^{*} B_{0} P_{\text {opt }}\right) \succeq B_{0} P_{\text {opt }}$. This implies $C \bar{A}^{*} \widetilde{S}_{0}^{\text {opt }} \succeq C \bar{A}^{*} B_{0} P_{\text {opt }}$, for an isotone mapping $C \bar{A}^{*}$. Therefore, the following equality holds $C \bar{A}^{*} B_{0} P_{o p t}=$ $C \bar{A}^{*} \widetilde{S}_{0}^{\text {opt }}$.

Proposition 5: Any matrix $\widetilde{S}_{0} \preceq \widetilde{S}_{0}^{o p t}$ can guarantee that matrix $P_{o p t}$ solves the DDP.

Proof: From Lemma 1, it is clear that equality (14) is achieved, i.e., the DDP is solved with $\widetilde{S}_{0}^{o p t}$ and $P_{\text {opt }}$, furthermore the following implication holds $\widetilde{S}_{0} \preceq \widetilde{S}_{0}^{\text {opt }} \Rightarrow$ $C \bar{A}^{*} \widetilde{S}_{0} \preceq C \bar{A}^{*} \widetilde{S}_{0}^{\text {opt }}=C \bar{A}^{*} B_{0} P_{\text {opt }}$, hence, equality (14) is achieved, i.e., the DDP is solvable by the optimal control $P_{\text {opt }}$ with any matrix $\widetilde{S}_{0} \preceq \widetilde{S}_{0}^{\text {opt }}$.

\section{Vi. A Manufacturing System Application}

The manufacturing system depicted in Fig. 1 is considered. Its frequency domain representation is given in Eq. (6). The transfer function between the output $Y(\gamma)$ and disturbance $Q(\gamma)$ and the input $U(\gamma)$, respectively, are

$$
\begin{aligned}
C \bar{A}^{*} S_{0} & =\left[\begin{array}{lll}
8\left(8 \gamma^{2}\right)^{*} & 7\left(7 \gamma^{2}\right)^{*} & 1\left(7 \gamma^{2}\right)^{*}
\end{array}\right] \\
C \bar{A}^{*} B_{0} & =\left[\begin{array}{ll}
17\left(8 \gamma^{2}\right)^{*} & 13\left(7 \gamma^{2}\right)^{*}
\end{array}\right]
\end{aligned}
$$

in which each component of these matrices is a periodic series. In this example, we have $\operatorname{Im} C \bar{A}^{*} S_{0} \subset \operatorname{Im} C \bar{A}^{*} B_{0}$, hence the DDP and MDDP are both solved by the controller filter $P_{\text {opt }}$. The example has been computed by using toolbox MinMaxGD, a C++ library allowing to handle periodic series as introduced in [9], and it can be noted that this library is also interfaced with Scilab and MATLAB.

The non-causal filter $P_{o p t}$ is by computing $C \bar{A}^{*} B_{0} \phi C \bar{A}^{*} S_{0}$ :

$$
\left(\begin{array}{ccc}
-9\left(8 \gamma^{2}\right)^{*} & \epsilon & \epsilon \\
-5\left(8 \gamma^{2}\right)^{*} & -6\left(7 \gamma^{2}\right)^{*} & -12\left(7 \gamma^{2}\right)^{*}
\end{array}\right)
$$

This prefilter $P_{o p t}$ is not causal because there are negative coefficients in the matrix. This non-causal prefilter, however, solves the MDDP and the DDP at the same time because $C \bar{A}^{*} B_{0} P_{\text {opt }}=C \bar{A}^{*} S_{0}$. If we take the canonical injection from the causal elements of $\overline{\mathbb{Z}}_{\max } \llbracket \gamma \rrbracket$ (denoted $\overline{\mathbb{Z}}_{\max } \llbracket \gamma \rrbracket^{+}$) in $\overline{\mathbb{Z}}_{\max } \llbracket \gamma \rrbracket$ (see [8] for details). Its residual is given by $\operatorname{Pr}\left(\bigoplus_{k \in \mathbb{Z}} s(k) \gamma^{k}\right)=\bigoplus_{k \in \mathbb{Z}} s_{+}(k) \gamma^{k}$ where

$$
s_{+}(k)=\left\{\begin{array}{l}
s(k) \text { if }(k, s(k)) \geq(0,0), \\
\varepsilon \text { otherwise. }
\end{array}\right.
$$

Therefore, the greatest causal prefilter is

$$
P_{o p t+}=\operatorname{Pr}\left(P_{o p t}\right)=\left(\begin{array}{ccc}
7 \gamma^{4}\left(8 \gamma^{2}\right)^{*} & \epsilon & \epsilon \\
3 \gamma^{2}\left(8 \gamma^{2}\right)^{*} & 1 \gamma^{2}\left(7 \gamma^{2}\right)^{*} & 2 \gamma^{4}\left(7 \gamma^{2}\right)^{*}
\end{array}\right) .
$$

The causal filter $P_{o p t+}$ for the MDDP does not solve the DDP because

$$
\begin{aligned}
C \bar{A}^{*} B_{0} P_{\text {opt+ }} & =\left[\begin{array}{lll}
16 \gamma^{2}\left(8 \gamma^{2}\right)^{*} & 14 \gamma^{2}\left(7 \gamma^{2}\right)^{*} & 15 \gamma^{4}\left(7 \gamma^{2}\right)^{*}
\end{array}\right] \\
& \neq C \bar{A}^{*} S_{0}
\end{aligned}
$$

We will find the new disturbance mapping $\widetilde{S}_{0}^{\text {opt }}=$ $C \bar{A}^{*} \phi\left(C \bar{A}^{*} B_{0} P_{o p t+}\right)$ as follows:

$$
\widetilde{S}_{0}^{\text {opt }}=\left[\begin{array}{ccc}
2 \gamma^{2}\left(8 \gamma^{2}\right)^{*} & \epsilon & \epsilon \\
8 \gamma^{2}\left(8 \gamma^{2}\right)^{*} & \epsilon & \epsilon \\
8 \gamma^{2}\left(8 \gamma^{2}\right)^{*} & 6 \gamma^{2}\left(7 \gamma^{2}\right)^{*} & 7 \gamma^{4}\left(7 \gamma^{2}\right)^{*} \\
9 \gamma^{2}\left(8 \gamma^{2}\right)^{*} & 7 \gamma^{2}\left(7 \gamma^{2}\right)^{*} & 8 \gamma^{4}\left(7 \gamma^{2}\right)^{*} \\
10 \gamma^{2}\left(8 \gamma^{2}\right)^{*} & 8 \gamma^{2}\left(7 \gamma^{2}\right)^{*} & 9 \gamma^{4}\left(7 \gamma^{2}\right)^{*} \\
15 \gamma^{2}\left(8 \gamma^{2}\right)^{*} & 13 \gamma^{2}\left(7 \gamma^{2}\right)^{*} & 14 \gamma^{4}\left(7 \gamma^{2}\right)^{*} \\
\gamma\left(8 \gamma^{2}\right)^{*} & \epsilon & \epsilon \\
7 \gamma\left(8 \gamma^{2}\right)^{*} & 5 \gamma\left(7 \gamma^{2}\right)^{*} & 6 \gamma^{3}\left(7 \gamma^{2}\right)^{*} \\
8 \gamma\left(8 \gamma^{2}\right)^{*} & 6 \gamma\left(7 \gamma^{2}\right)^{*} & 7 \gamma^{3}\left(7 \gamma^{2}\right)^{*}
\end{array}\right]
$$

According to Proposition 5, for any matrix $\widetilde{S}_{0} \preceq \widetilde{S}_{0}^{o p t}$, both the DDP and the MDDP are solved by considering the same causal filter $P_{o p t+}$. For example, let $\widetilde{S}_{0}$ be a matrix with all its entries equal to $\varepsilon$ except for the non null ones of matrix 
$S_{0}$, i.e., the following one $\widetilde{S}_{0}(2,1)=\widetilde{S}_{0}^{o p t}(2,1), \widetilde{S}_{0}(4,2)=$ $\widetilde{S}_{0}^{o p t}(4,2), \widetilde{S}_{0}(6,3)=\widetilde{S}_{0}^{o p t}(6,3)$. This matrix will be such that the DDP is solvable with $P_{\text {opt+ }}$. Practically, this means that transition $x_{2}$, which was initially directly disturbed by the uncontrollable input $q_{1}$ in the original model, has to be disturbed by $8 \gamma^{2}\left(8 \gamma^{2}\right)^{*} q_{1}$, i.e. the state equation in the event domain is $x_{2}(k)=6 x_{1}(k) \oplus 8 \gamma^{2}\left(8 \gamma^{2}\right)^{*} q_{1}(k)$. This equation can be realized as follows in the time domain $x_{2}(t)=\min \left(x_{1}(t-\right.$ $\left.6), w_{1}(t)\right)$ with $w_{1}(t)=\min \left(2+q_{1}(t-8), 2+w_{1}(t-8)\right)$. The term $2+q_{1}(t-8)$ means that disturbance has to be measured and that two tokens have to be initially present between $q_{1}$ and $x_{2}$. If you consider $q_{1}$ as an uncontrollable component supply input, these two tokens depict the minimal stock you need to be able to solve the DDP. More generally, $\widetilde{S}_{0}^{o p t}$ represents the optimal way you need to modify for the system in order to be able to reject disturbances. In a manufacturing system, it can represent the minimal initial stock of raw parts you need to be robust according to machine breakdown or any malfunctions in exogenous component supply.

\section{CONCLUSION}

This paper defines a new concept of the MDDP and establishes the optimal controller to solve the MDDP. Moreover, a necessary and sufficient condition is established such that the optimal controller for the MDDP can solve the DDP for max-plus linear systems. When the necessary and sufficient condition is not satisfied, a new disturbance mapping is developed in order to guarantee the solvability of the DDP using the same optimal controller for the MDDP. This paper only deals with open-loop controllers for the MDDP, the next research problem is to establish the optimal state feedback controllers and the output feedback controllers for the MDDP, and study the relationships between these solutions to the DDP and the MDDP.

\section{REFERENCES}

[1] Baccelli, F., Cohen, G., Olsder, G.J., \& Quadrat, J.-P. (1992) Synchronization and Linearity: An Algebra for Discrete Event Systems. New York: John Wiley and Sons. http://www-rocq. inria.fr/ metalau/cohen/SED/SED1-book.html

[2] Cohen, G., Gaubert, S., \& Quadrat, J.-P. (1999) Max-plus algebra and system theory: where we are and where to go now. Annual Reviews in Control, 23, 207-219.

[3] Cohen, G. (1995) Théorie algébrique des systèmes à événements discrets, Polycopié de cours donné àl'INRIA.

[4] Cohen, G., Gaubert, S., \& Quadrat, J.-P. (1996) Kernels, images, and projections in dioids, Proceedings of WODES'96, Edinburgh.http: //www-rocq.inria.fr/metalau/quadrat/kernel.pdf

[5] Cohen, G., Gaubert, S., \& Quadrat, J.-P. (1997) Linear projectors in the max-plus algebra. The 5th IEEE-Mediterranean Conference, Paphos, Cyprus. http://www-rocq.inria.fr/metalau/ quadrat/projector.pdf

[6] Cohen, G., Gaubert, S., \& Quadrat, J.-P. (1999) Max-plus algebra and system theory: where we are and where to go now. Annual Reviews in Control, 23, 207-219.

[7] Cohen, G., Gaubert, S., \& Quadrat, J.-P. (2006) Projection and Aggregation in Maxplus Algebra, Current Trends in Nonlinear Systems and Control, in Honor of Peter Kokotovic and Turi Nicosia, Boston: Birkhäuser. http://www-rocq.inria.fr/metalau/ quadrat/Rome.pdf

[8] Cottenceau, B., Hardouin, L., Boimond, J.-L., \& Ferrier,J.-L. (2001) Model reference control for timed event graphs in dioid. Automatica, 37, 1451-1458.
[9] Cottenceau, B., Hardouin, Lhommeau, M. \& Boimond, J.-L. (2000) Data processing tool for calculation in dioid, The 5th Workshop on Discrete Event Systems, Ghent, Belgium, http://www.istia. univ-angers.fr/ hardouin/outils.html

[10] Di Loreto, M., Gaubert, S., Katz, R. \& Loiseau, J-J, (2010) Duality between invariant spaces for max-plus linear discrete event systems, Siam Journal on Control and Optimization 48, 5606-5628, http: // fr.arXiv.org/abs/0901.2915.

[11] Golan, J.S. (1999) Semirings and Their Applications. Boston: Kluwer Academic Publishers.

[12] Hardouin, L., Maia, C.A., Cottenceau, B, \& Lhommeau, M. (2010) Observer Design for (max,plus)-Linear Systems, IEEE Transactions on Automatic Control, 55, 2, 538-543.

[13] Hardouin, L., Lhommeau, M. \& Shang,Y. (2011) Towards Geometric Control of Max-Plus Linear Systems with Applications to Manufacturing Systems, The 50th IEEE Conference on Decision and Control and European Control Conference, 1149-1154.

[14] De Jong, H. (2002) Modeling and simulation of genetic regulatory systems: a literature review. J. Computational Biology, 9, 1, 67-103.

[15] Katz, R.D. (2007) Max-plus (A,B)-invariant spaces and control of timed discrete-event systems, IEEE Trans. Auto. Control, 52, 2, 229241.

[16] Le Boudec, J.-Y. \& Thiran, P. (2002) Network Calculus. New York: Springer-Verlag.

[17] Lhommeau, M., Hardouin, L., \& Cottenceau, B. (2002) Disturbance decoupling of timed event graphs by output feedback controller, The 6th Workshop on Discrete Event Systems Zaragoza, Spain, http:// www. istia.univ-angers.fr/ hardouin/Wodes 02 .pdf

[18] Lhommeau, M., Hardouin, L., \& Cottenceau, B. (2003) Optimal control for $(\max ,+)$-linear systems in the presence of disturbances, Positive Systems: Theory and Applications, POSTA, Springer LNCIS 294, 47-54.

[19] Maia, C.A, Hardouin,L., Santos-Mendes, R., \& Cottenceau, B.(2005) On the Model reference control for max-plus linear systems. The 44th IEEE Conference on Decision and Control, 7799-7803.

[20] Maia, C.A, Andrade, C.R. \& Hardouin, L. (2011) On the control of max-plus linear system subject to state restriction. Automatica, 47-5, 988-992.

[21] Prou, J.-M., \& Wagneur, E. (1999) Controllability in the max-algebra. KYBERNETIKA. 35, 1, 13-24.

[22] Wonham, W.M. (1979) Linear Multivariable Control: A Geometric Approach. New York: Springer-Verlag. 